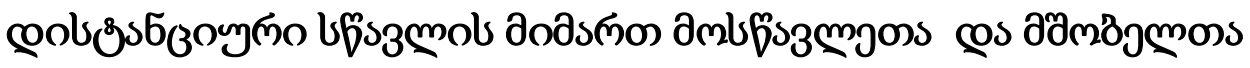

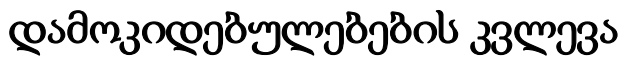

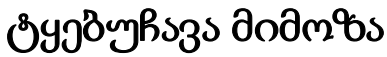

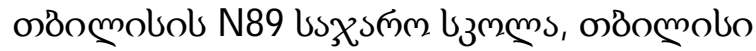

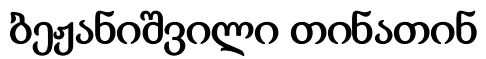

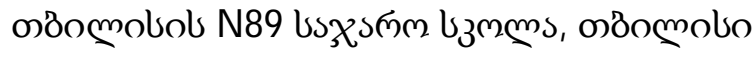 \\ https://doi.org/10.52340/idw .2021.544
}

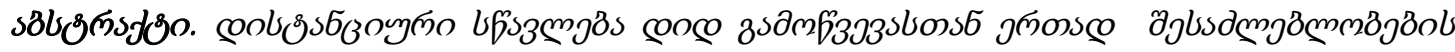

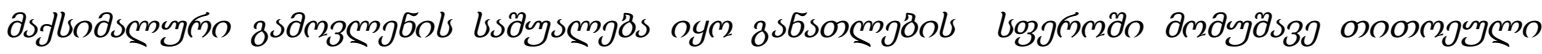

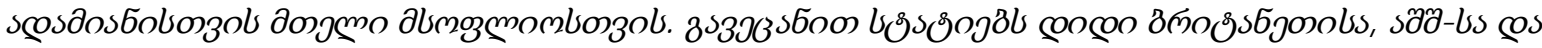

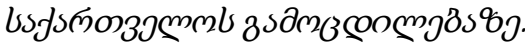

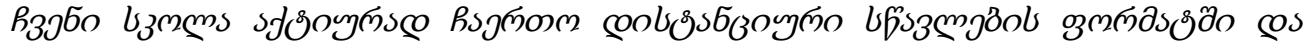

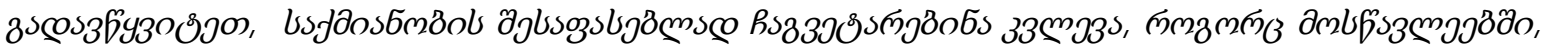

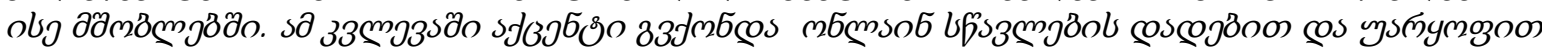

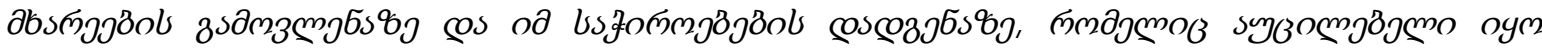

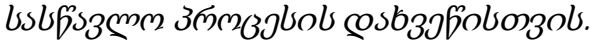

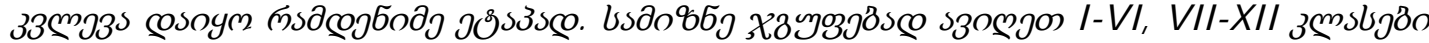

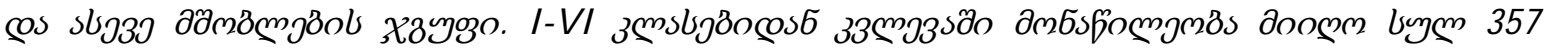

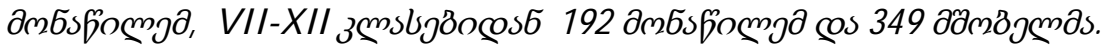

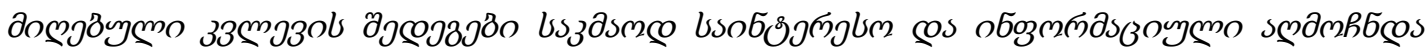

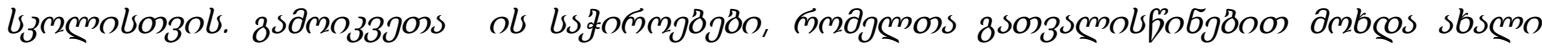

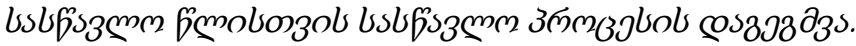

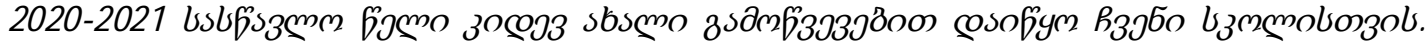

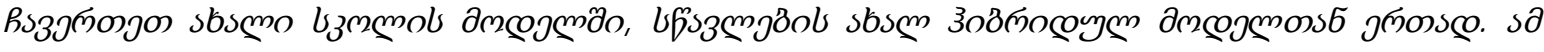

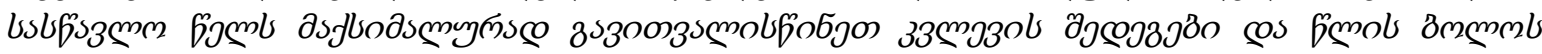

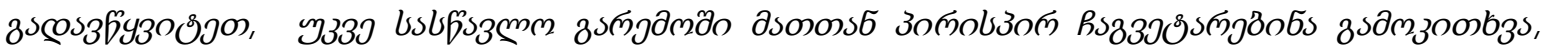

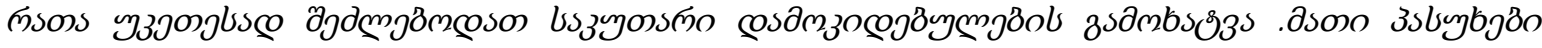

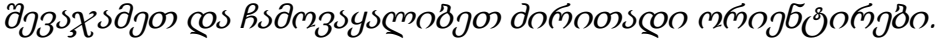

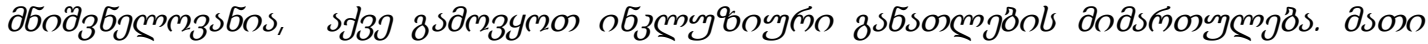

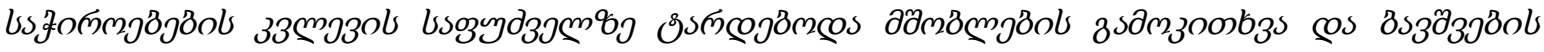

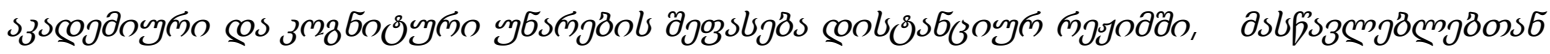

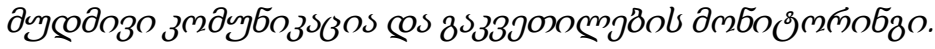

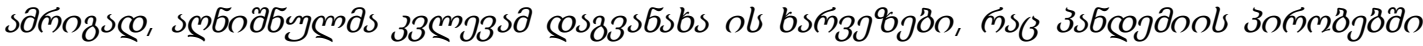

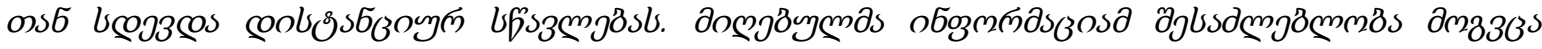

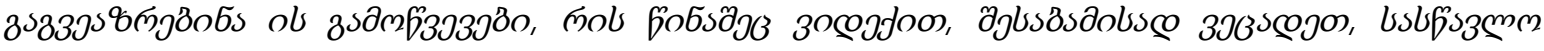
зпмиј

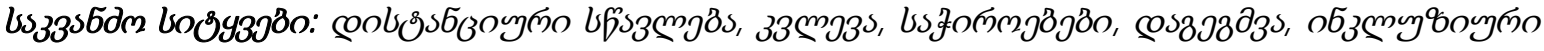

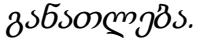

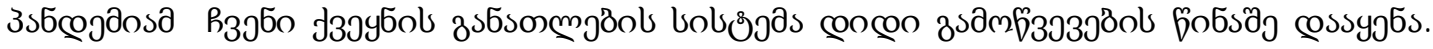

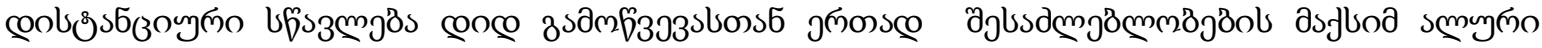

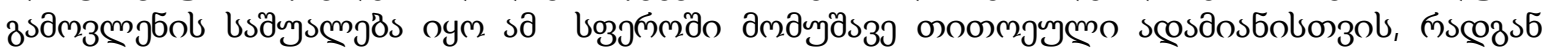




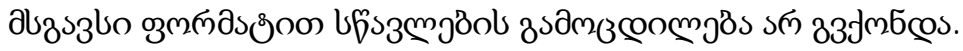

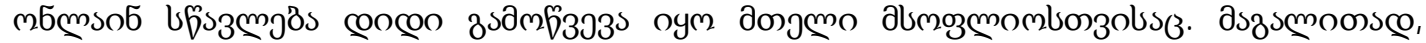

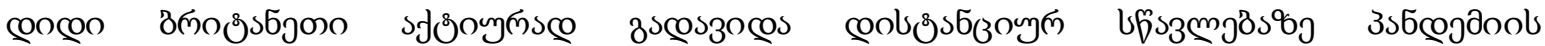

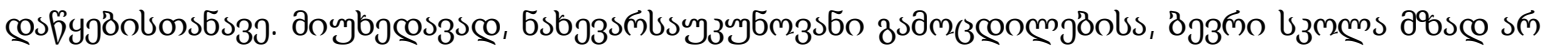

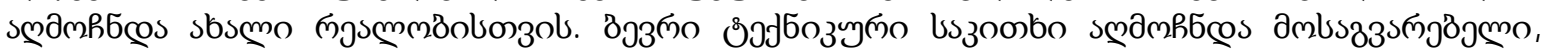

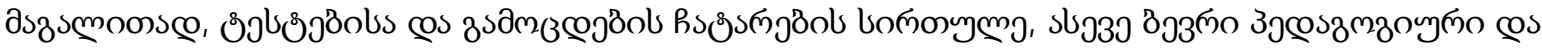

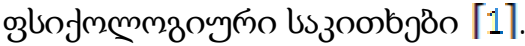

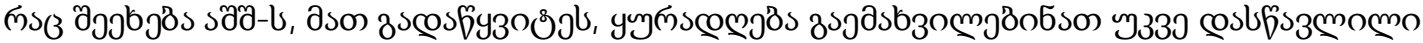

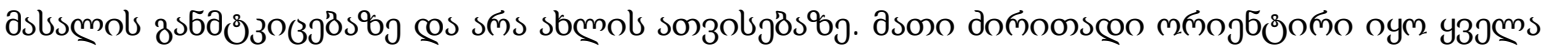

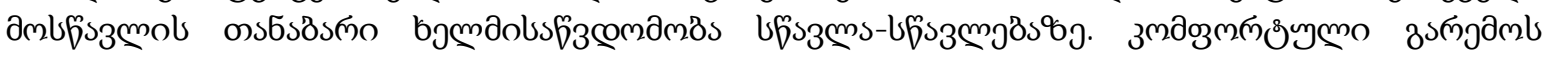

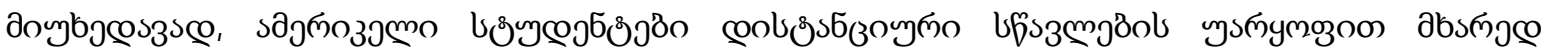

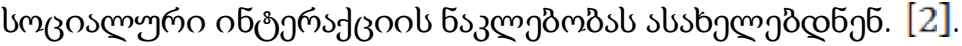

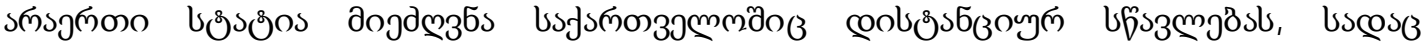

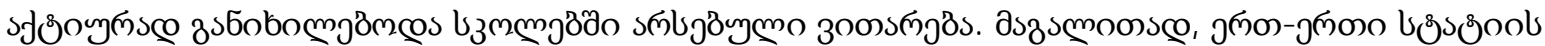

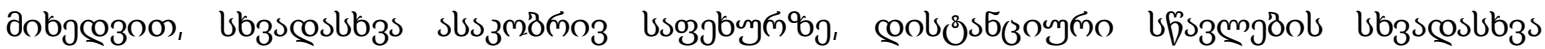

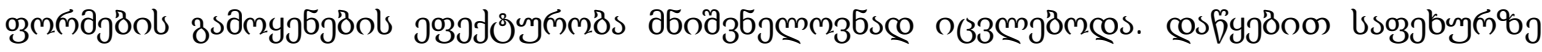

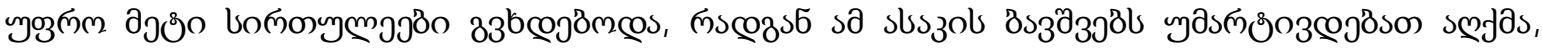

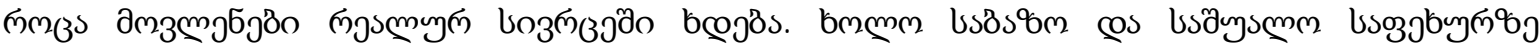

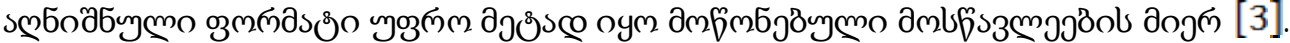

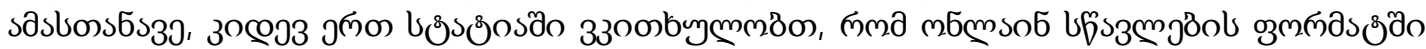

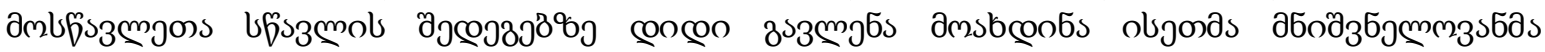

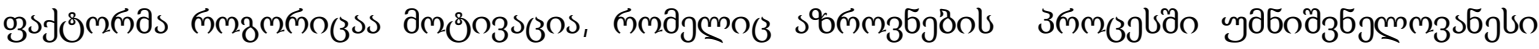

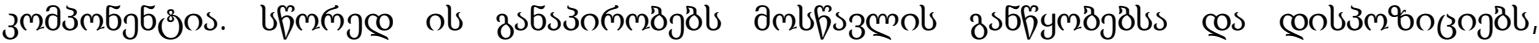

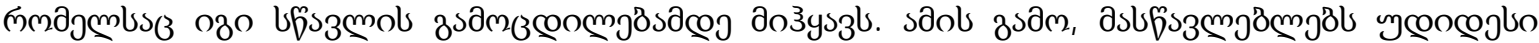

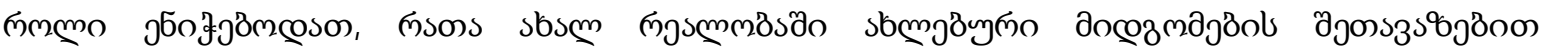

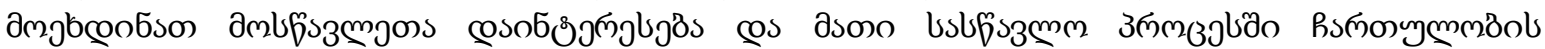

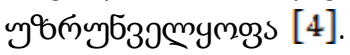

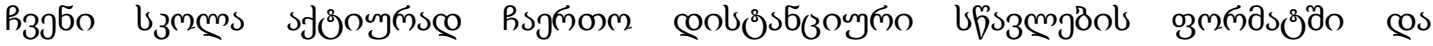

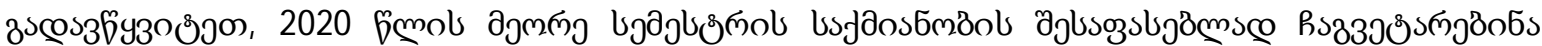

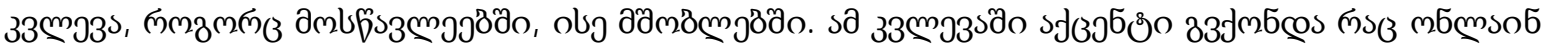

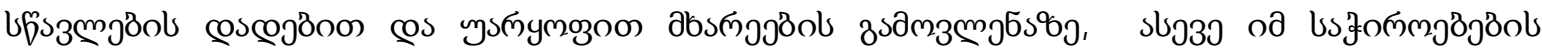

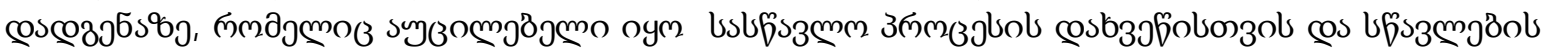

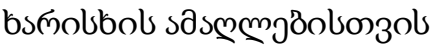

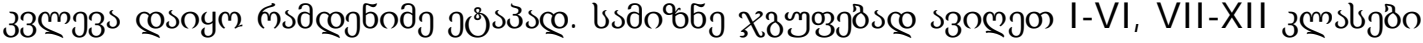

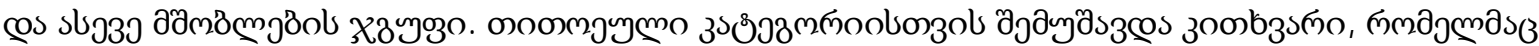

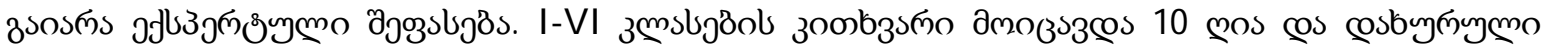

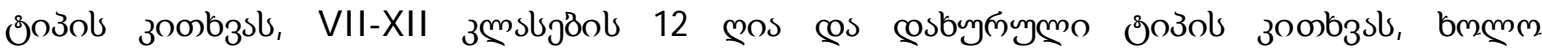

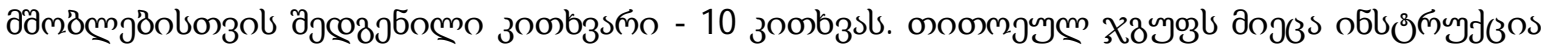

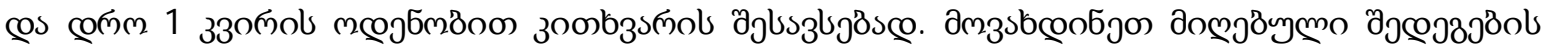

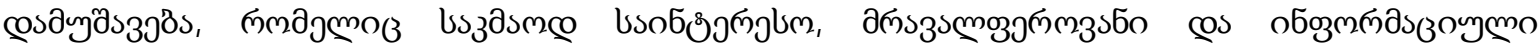

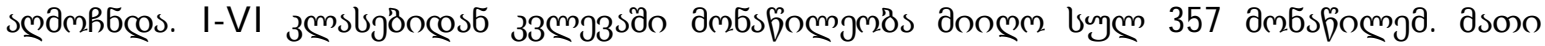

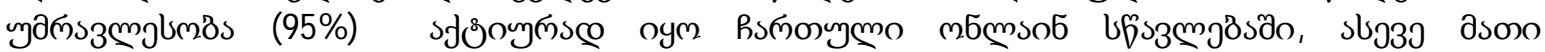

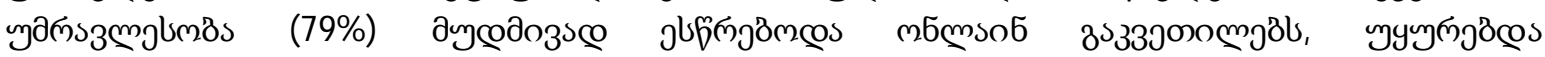

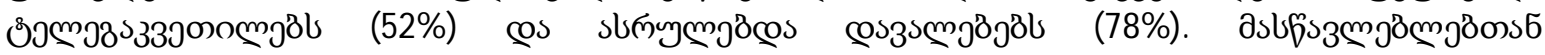

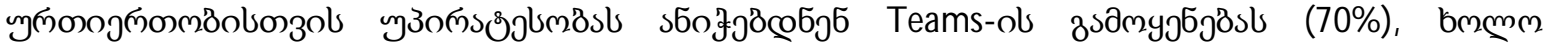

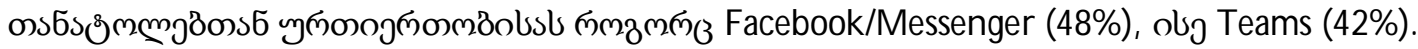

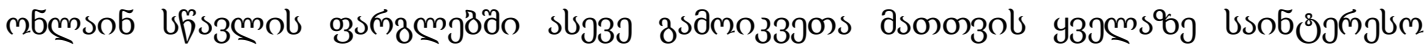

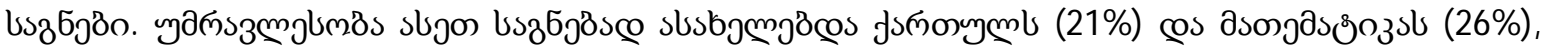

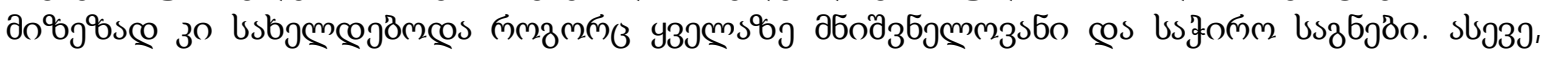

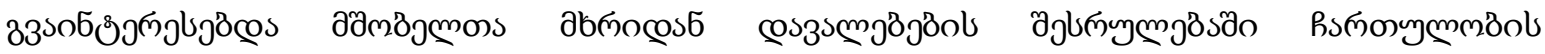




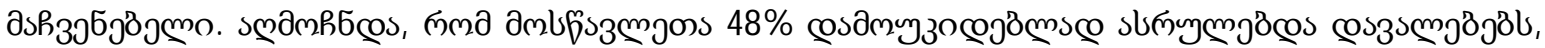

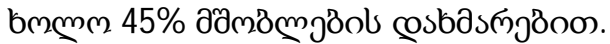

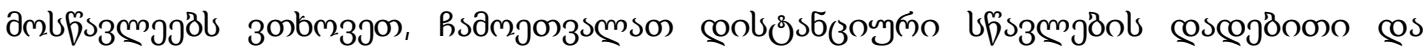

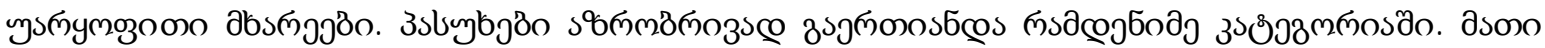

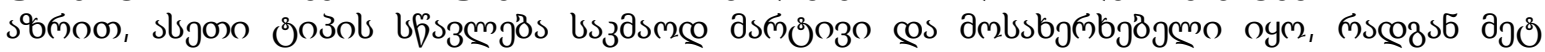

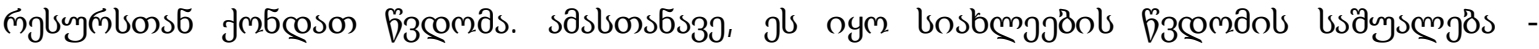

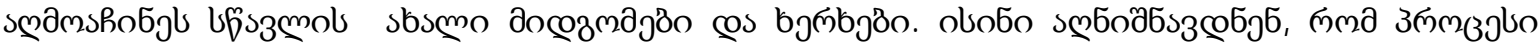

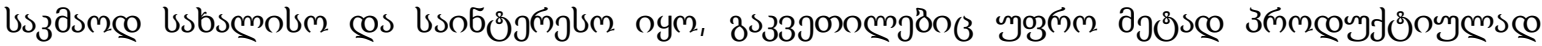

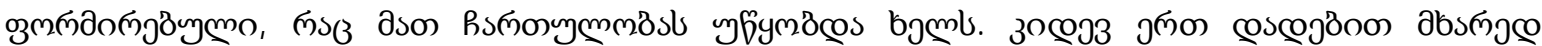

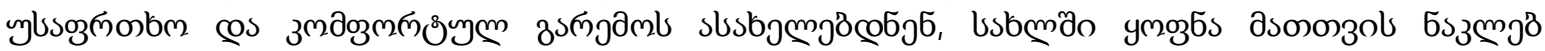

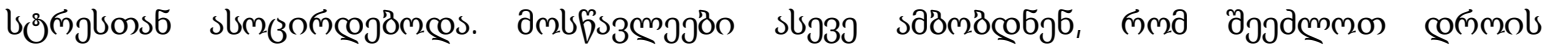

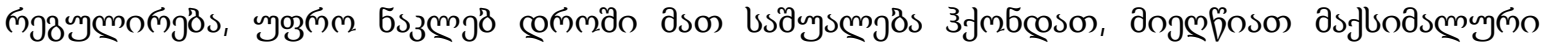

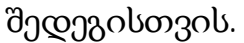

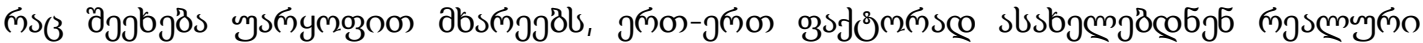

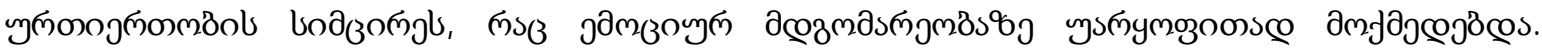

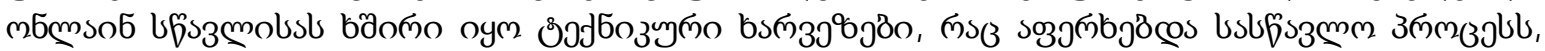

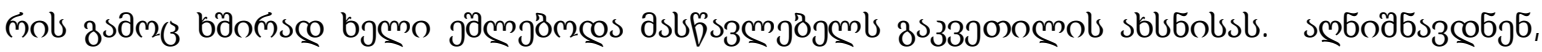

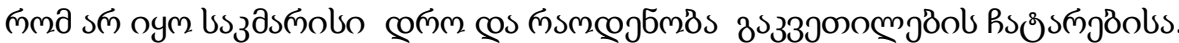

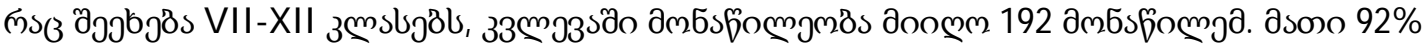

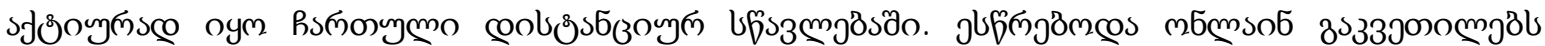

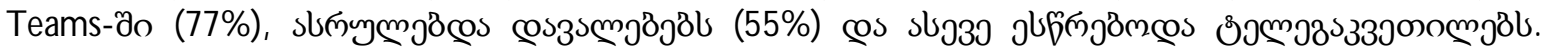

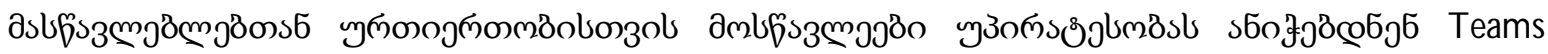

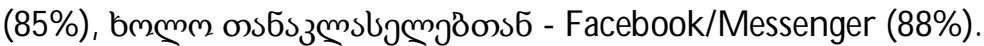

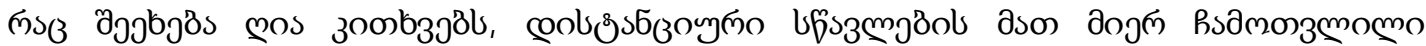

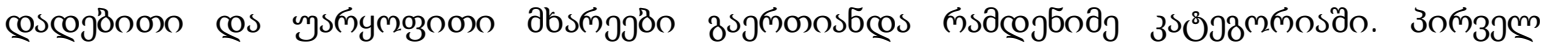

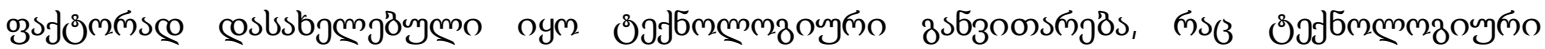

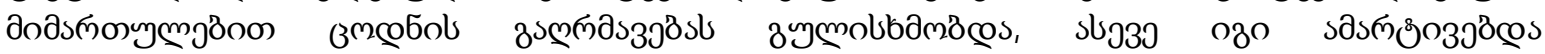

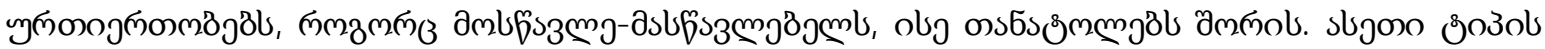

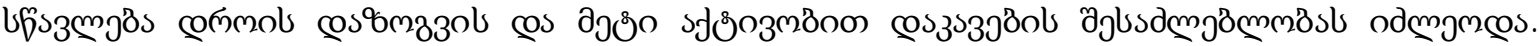

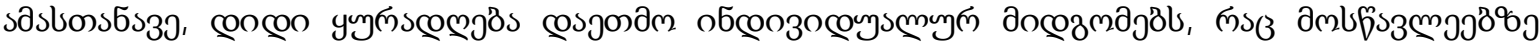

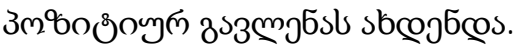

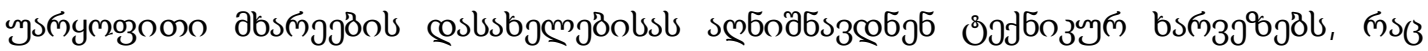

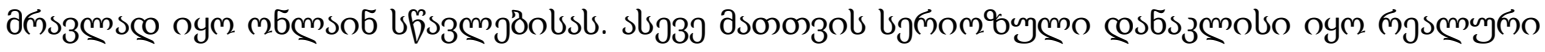

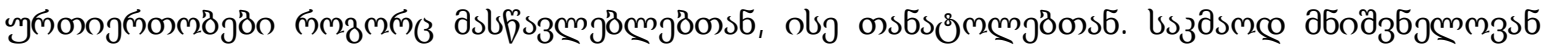

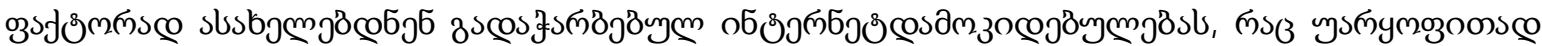

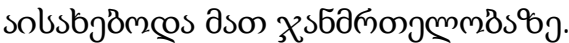

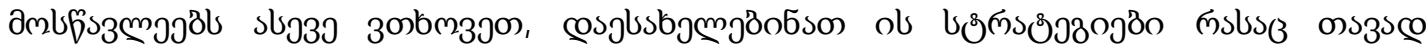

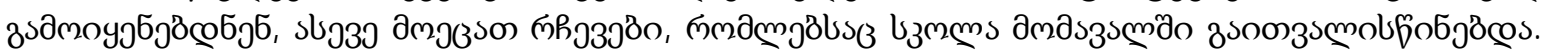

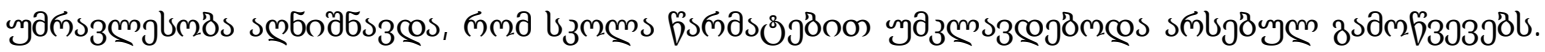

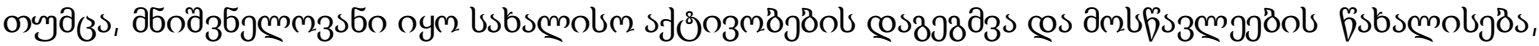

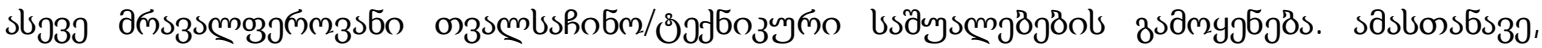

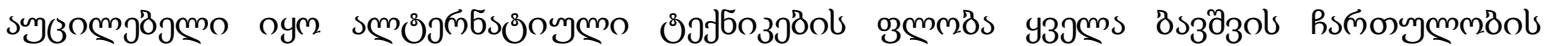

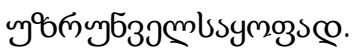

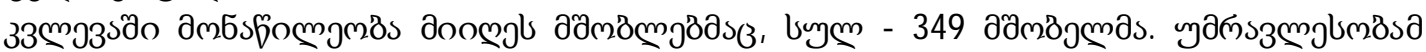

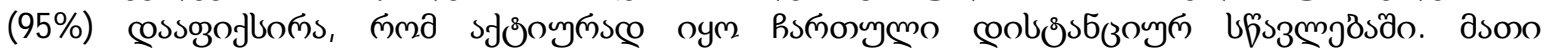

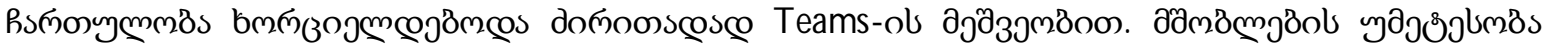

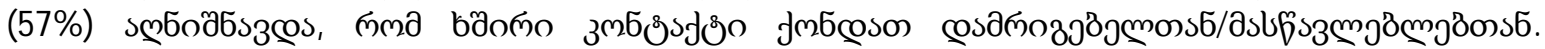

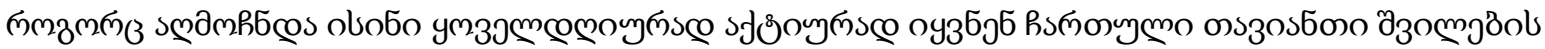

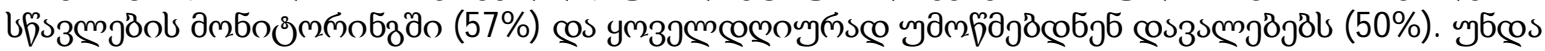

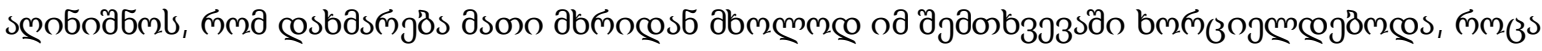




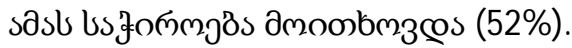

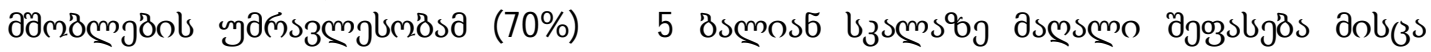

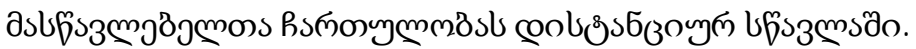

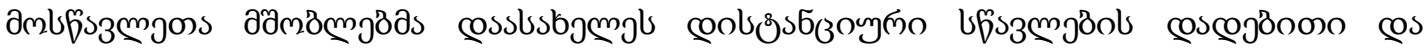

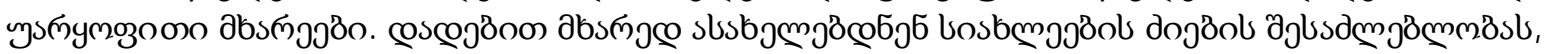

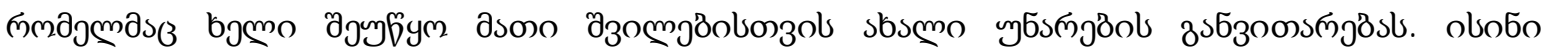

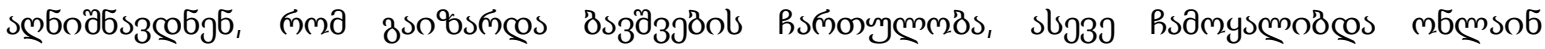

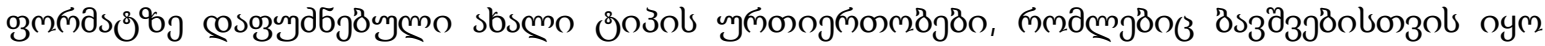

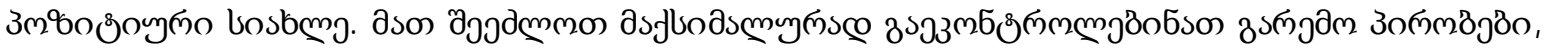

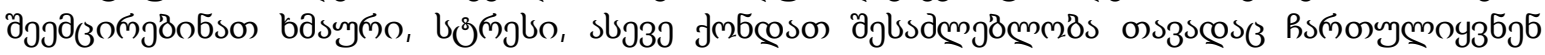

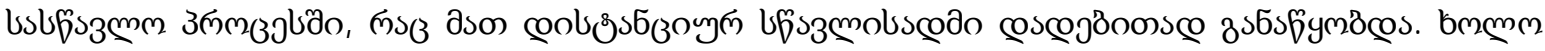

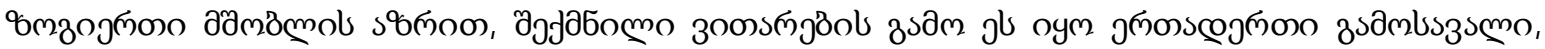

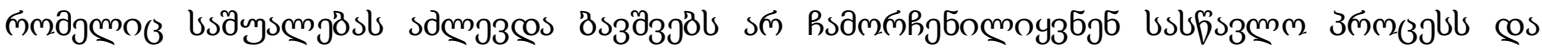

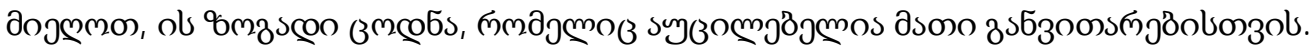

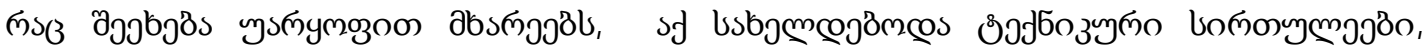

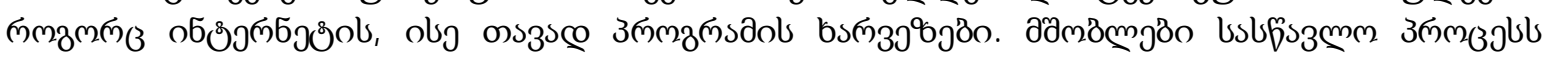

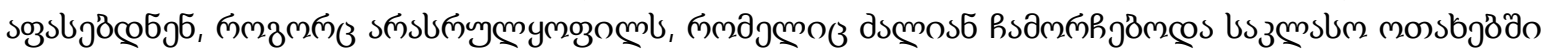

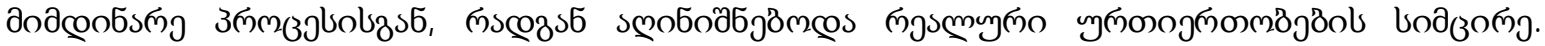

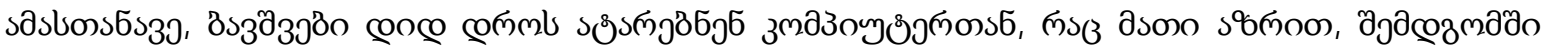

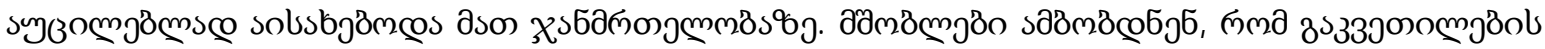

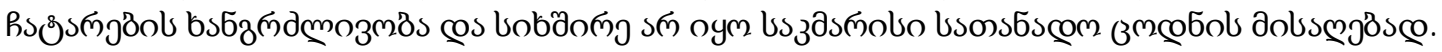

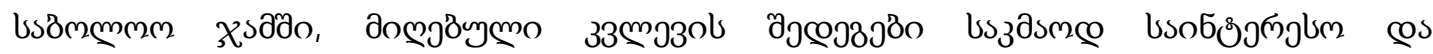

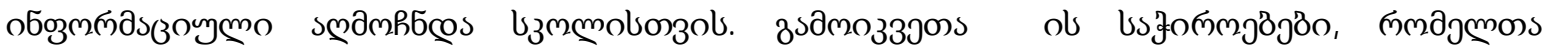

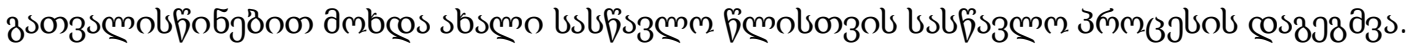

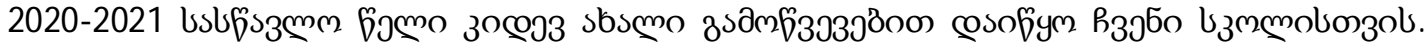

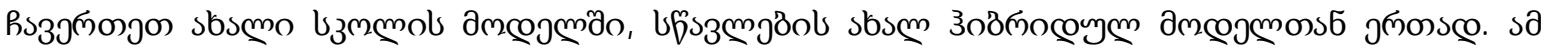

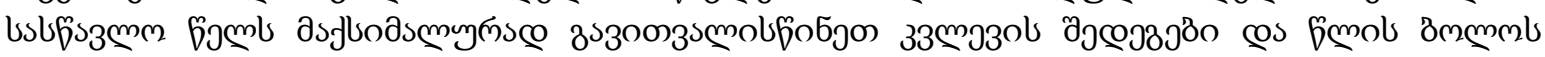

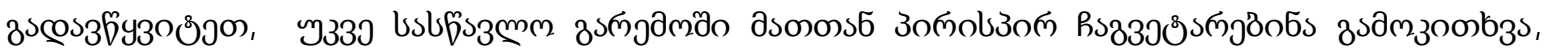

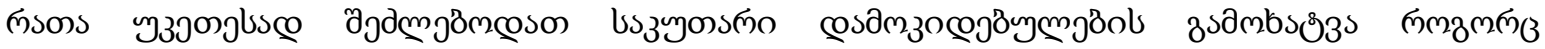

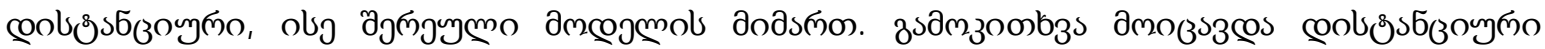

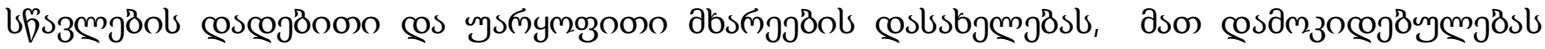

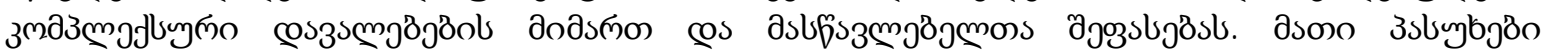

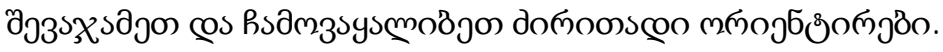

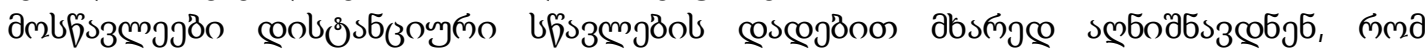

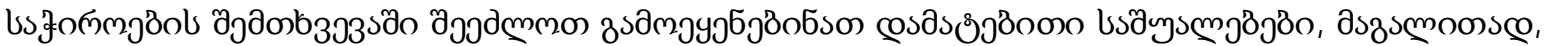

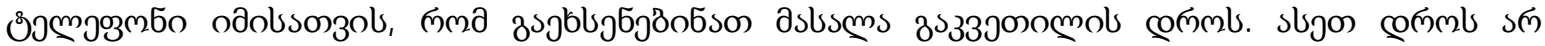

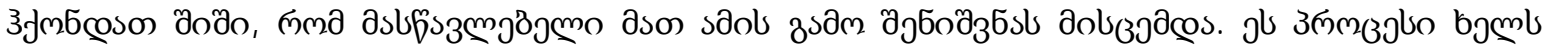

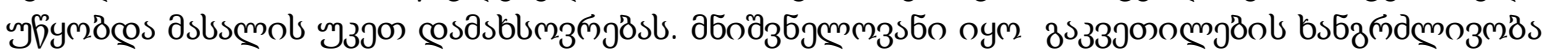

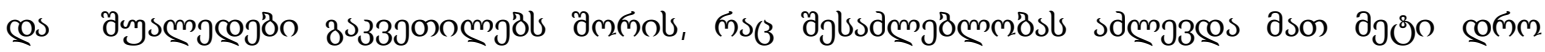

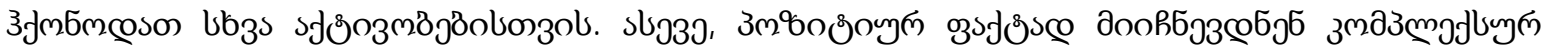

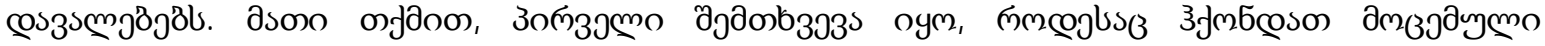

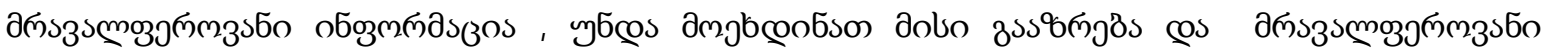

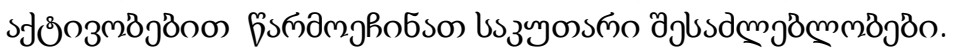

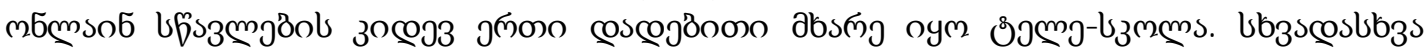

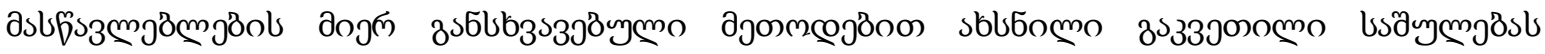

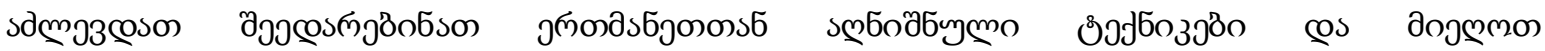

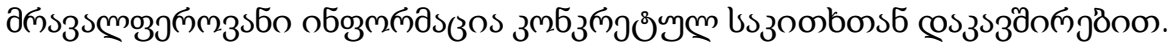

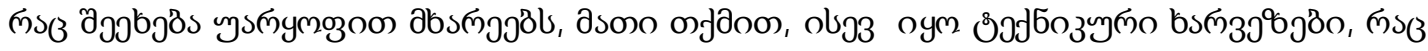

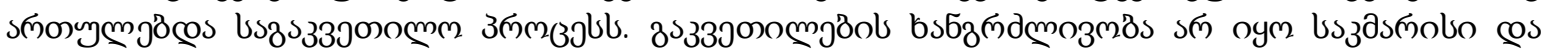

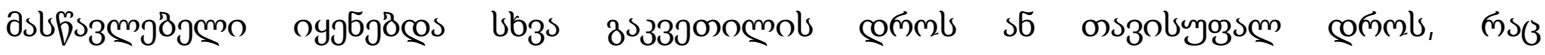




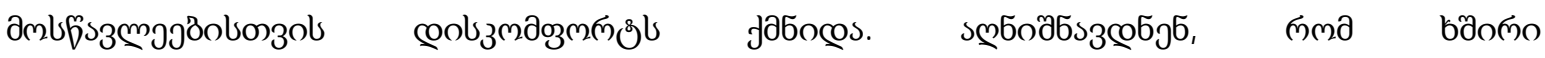

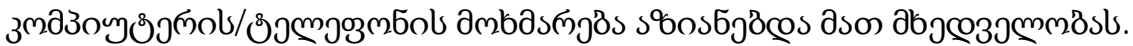

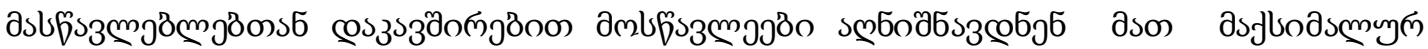

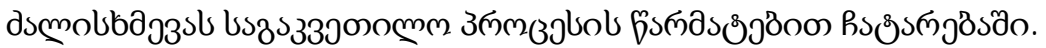

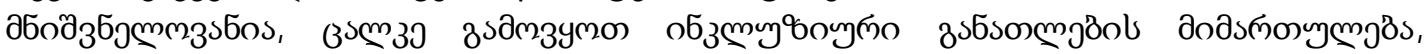

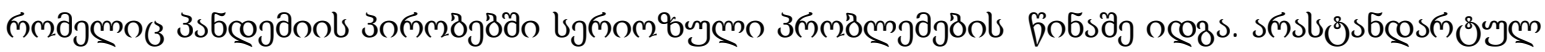

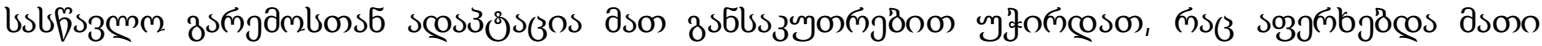

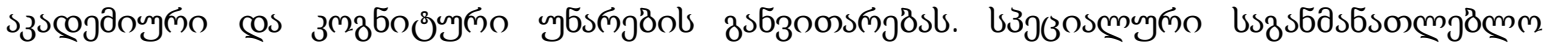

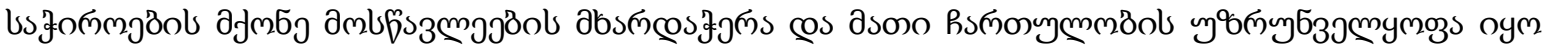

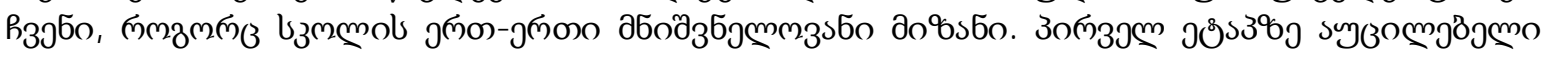

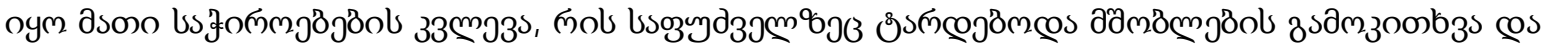

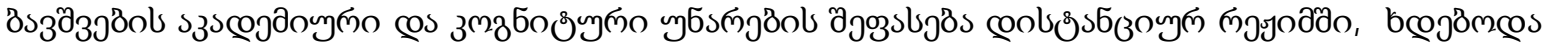

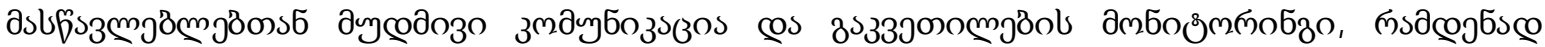

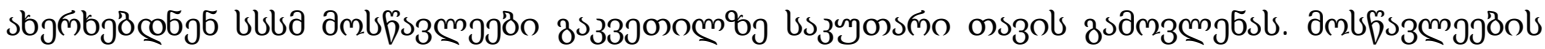

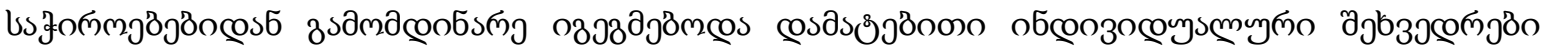

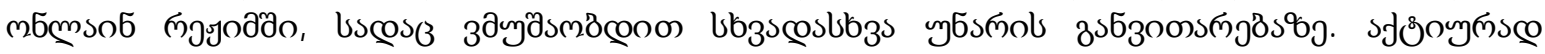

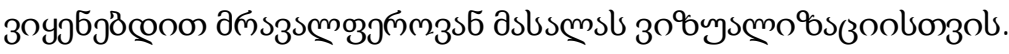

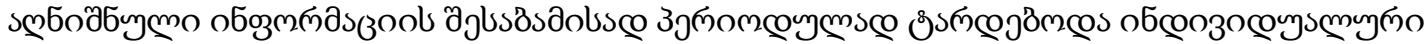

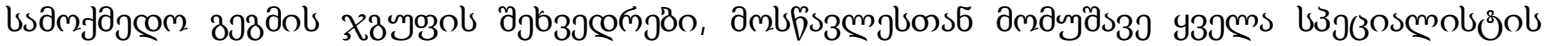

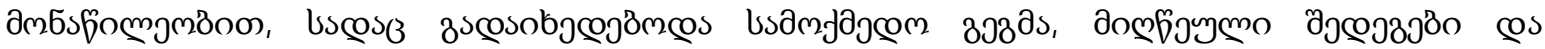

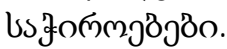

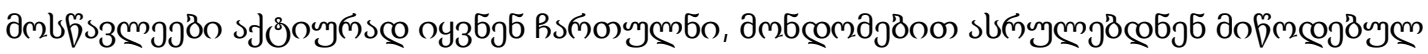

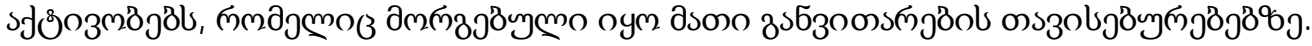

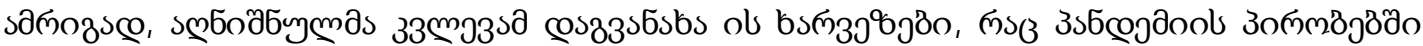

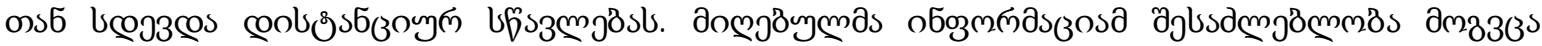

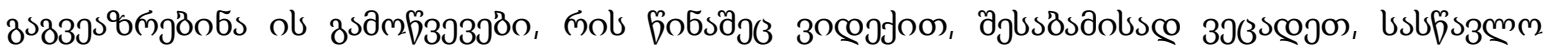

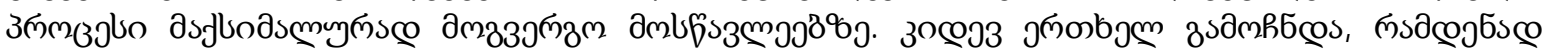

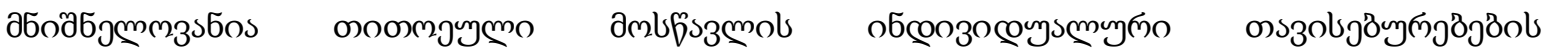

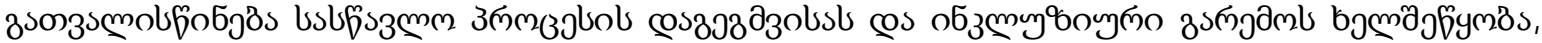

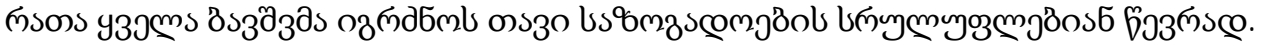

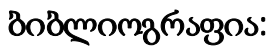

1. 3iysmcoos6o, T. G. (2020). H ow to study at home during coronavirus (26.03.2020).

2. Eye, N. (2020). Online Learning in the Time of COVID-19: W hat are the Pros and Cons. Scholarship America.

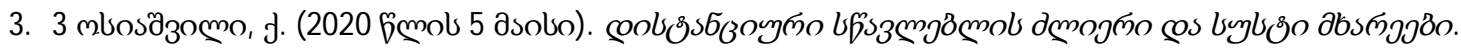

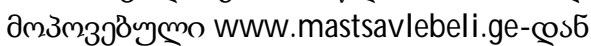

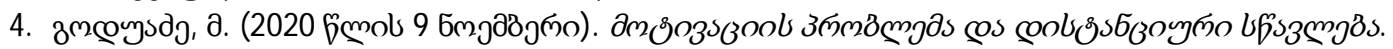

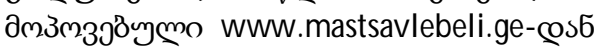




\title{
A Study of Students and Parents' Attitudes towards Distance Learning
}

\author{
Bezhanishvili Tinatin \\ Tbilisi Public School N89, Tbilisi \\ Tkebuchava Mimoza \\ Tbilisi Public School N89, Tbilisi
}

\begin{abstract}
Distance learning was a mean of maximizing opportunities with a big challenge for the people in the world who works in the education space. We read the articles about experience of Great Britain, USA and Georgia.

Our school was involved in distance learning actively and we conducted a survey of as schoolchildren as parents in order to value our activity. In this study, we focused on identifying the pros and cons of online learning that were necessary to refine the learning process.

The research began in several stages. We took $1^{\text {st }}-6^{\text {th }}, 7^{\text {th }}-12^{\text {th }}$ grades as target groups and parents' group too. 357 students participated in the study from $1^{\text {st }}$ and $6^{\text {th }}$ grades and 192 participants and 349 parents participated from $7^{\text {th }}$ and $12^{\text {th }}$ grades.

The results of research were quite interesting and informative for the school. The study identified the needs with we planned the new learining process.

2020-2021 has also started with new challenges for our school. We were involved in a new school model, along with a new hybrid model of teaching. We took into account research results and at the end of the year decided to conduct a face-to-face survey in a learning environment in order to better express their attitudes. We summarized their answers and formed the main orientaries.

It is important to emphasize the direction of inclusive education. According the research, we assessed children's academic and cognitive skills online and interviewed parents and teachers, made lessons' monitoring...

Thus, this research showed the faults that accompanied distance learning during the pandemic period. The information that we received enabled us to understand the challenges we faced, accordingly, we tried to adapt the learning process to the student as much as possible.
\end{abstract}

Keywords: distance learning, research, needs, planning, inclusive education. 